\title{
DEPRESSÃO EM PACIENTES COM EPILEPSIA REFRATÁRIA DO LOBO TEMPORAL MESIAL
}

\author{
Juliana Unis Castan \\ Fernanda Rohrsetzer Cunegatto \\ (Hospital de Clínicas de Porto Alegre - RS)
}

\begin{abstract}
Resumo
Epilepsia é um transtorno neurológico crônico caracterizado por crises convulsivas recorrentes, o qual tem sido associado com comorbidades psiquiátricas, especialmente depressão. Objetivo: verificar níveis de sintomas de depressão em pacientes com Epilepsia Refratária do Lobo Temporal (ELT), internados para videomonitorização em um hospital geral. Método: trata-se de um estudo exploratório, transversal e de natureza quantitativa. Níveis de sintomas de depressão foram obtidos através da Escala Beck de Depressão (BDI). A amostra constituiu-se de pacientes internados para videomonitorização da epilepsia, entre os meses de janeiro de 2016 e dezembro de 2017. Resultados: 63\% da amostra obteve sintomas de depressão classificados em níveis Mínimo ou Leve; 32\% em nível Moderado e 5\% em nível Grave. Conclusão: Os níveis de sintomas depressivos da amostra deste estudo foram consideravelmente superiores à prevalência mundial e brasileira. Esta realidade aponta para a importância de um diagnóstico em tempo hábil com o objetivo de obter tratamento adequado para esta população.
\end{abstract}

Palavras-chave: Depressão; BDI, Epilepsia Refratária; Epilepsia do lobo temporal.

\section{Abstract \\ Depression in Patients with Refractory Epilepsy Mesial Temporal Lobe}

Epilepsy is a chronic neurological disorder characterized by recurring epileptic seizures. It has been associated with psychiatric disorders, mainly depression. Objective: to verify levels of symptoms of depression in patients with Refractory Temporal Lobe Epilepsy (ELT) hospitalized for videomonitoring in a general hospital. Methods: This is an exploratory, crosssectional and quantitative study. Levels of symptoms of depression were measured by Beck Depression Inventory (BDI). The sample consisted of patients admitted to a general hospital for videomonitoring between January 2016 and December 2017. Results: 63\% of the sample has symptoms of depression classified as Minimum or Mild; 32\% Moderate and 5\% Severe. Conclusion: Depressive symptoms of the sample were higher than that of the general population, both worldwide and Brazilian. This points out to the importance of a timely diagnosis in order to obtain adequate treatment for this population.

Keywords: Depression; BDI; Refractory epilepsy; Epilepsy of the temporal lobe. 


\section{Introdução}

Epilepsia é uma condição cerebral caracterizada pela predisposição a crises convulsivas e por seus efeitos neurobiológicos, cognitivos, psicológicos e sociais. Crises convulsivas são manifestações clínicas abruptas, resultado de descarga elétrica anormal, súbita e transitória. Possuem diversas formas de manifestações clínicas, incluindo motora, sensitiva, psíquicas, comportamental ou autônoma, dependendo da região cerebral da descarga e da propagação (Fisher et al., 2005). Aproximadamente 65 milhões de pessoas no mundo sofrem de epilepsia, considerada a doença neurológica mais prevalente (Laxer et al., 2014).

O tratamento base da epilepsia constitui-se no uso diário de medicações como forma de controlar a ocorrência das crises. No entanto, cerca de um terço dos pacientes com epilepsia segue com convulsões recorrentes, apesar do uso de fármacos adequados e boa adesão ao tratamento, sendo esta classificada como epilepsia refratária (Freitas-Lima et al., 2013). A epilepsia refratária pode ser definida como a resistência a, pelo menos, duas drogas antiepilépticas utilizadas adequadamente, sem estabelecimento do controle das crises (Kwan et al., 2010).

A Epilepsia do Lobo Temporal (ELT) é a de maior prevalência em adultos, sendo responsável por $40 \%$ dos casos de epilepsia focal nesta faixa etária (AndradeValença, Valença, Velasco \& Leite, 2006). Destas, em torno de $30-40 \%$ permanecem refratárias ao tratamento farmacológico (Gonçalves et al., 2017), sendo o tratamento cirúrgico, que visa a ressecção do foco epileptogênico, uma alternativa no combate às crises e às decorrentes perdas neuronais (Engel et al., 2012; Valeriano-Lorenzo et al., 2016). A investigação pré-operatória é composta por monitorização e registro de crises através de vídeo eletroencefalograma (VEEG), exames de neuroimagem e testagem neuropsicológica.

A avaliação neuropsicológica permite entender o impacto funcional da 
epilepsia e seu tratamento. Através de entrevistas, observações e testes psicométricos, tem por objetivo auxiliar na localização e lateralização da região epileptogênica, identificar reserva e deterioro em funções específicas de cada hemisfério cerebral e estabelecer uma linha de base para futuras avaliações (Fuentes, Malloy-Diniz, Camargo \& Cosenza, 2014; Ramos \& Hamdan, 2016). Avaliação de fatores psicossociais, incluindo o humor e o funcionamento psicológico do paciente, assim como questões familiares e sociais, permite uma visão abrangente do impacto da doença e de seus efeitos, permitindo acompanhamento e assessoramento na tomada de decisão quanto a possíveis tratamentos, inclusive o cirúrgico (Baxendale \& Thompson, 2010).

Cerca de 20 a $50 \%$ dos pacientes com epilepsia apresentam transtorno de humor como comorbidade psiquiátrica, principalmente pessoas com controle precário das crises (Kanner, Byrne, Chicharro, Wuu \& Frey, 2009). A depressão é o transtorno psiquiátrico mais comum em pacientes com epilepsia refratária (Scévola et al, 2017). A associação entre epilepsia e depressão ocorre possivelmente tanto por fatores intrínsecos, como questões biológicas/fisiológicas, como fatores extrínsecos, como aspectos psicológicos e sociais. Estudos sugerem que há uma possível relação bidirecional entre depressão e epilepsia, com caminhos patogênicos comuns (Kanner et al., 2012; Orjuela-Rojas, Martinez-Juárez, RuizChow \& Crail-Melendez, 2015; Scévola et al. 2017; Torres et al., 2017). Desta forma, ser portador de epilepsia aumentaria o risco para um possível quadro de depressão, ao passo que pacientes com diagnóstico prévio de transtorno depressivo apresentariam risco aumentado para epilepsia. Somado a isso, fatores psicossociais, como estigma social, superproteção familiar, baixo nível socioeconômico, desemprego e cronicidade da doença, também contribuiriam para quadros depressivos. 
Sintomas de depressão não tratados podem dificultar controle das crises (Dias et al., 2010; Kanner, Byrne, Chicharro, Wuu \& Frey, 2009), além de terem um impacto na qualidade de vida e na recuperação dos pacientes submetidos à cirurgia. Sendo assim, este trabalho tem por objetivo verificar níveis de depressão, através da escala Beck de Depressão (BDI), em pacientes com Epilepsia Refratária do Lobo Temporal, internados para videomonitorização em leitos da internação na Unidade de Epilepsia em um hospital geral universitário no período de janeiro de 2016 e dezembro de 2017.

\section{Método}

Trata-se de um estudo exploratório, transversal e de natureza quantitativa. De forma retrospectiva, realizou-se um levantamento de informações demográficas e do nível de sintomas de depressão, de acordo com a escala Beck de Depressão (BDI). A amostra constituiu-se de pacientes internados para exploração diagnóstica da ELT refratária à medicação, submetidos à bateria de avaliação neuropsicológica no período de janeiro de 2016 a dezembro de 2017. A pesquisa foi realizada em um hospital universitário da região sul do Brasil que conta com dois leitos equipados para monitorização através de VEEG.

\section{Instrumentos}

O BDI é um instrumento de autoavaliação da gravidade de sintomas depressivos. A escala é constituída por 21 itens e pode ser autoaplicada ou aplicada por um entrevistador. Cada item possui uma variação de intensidade de 0 a 3 em relação às atitudes e sintomas. Os pontos de corte recomendados para a versão do BDI em português são: 0 a 11 mínimo; 12 a 19 leve; 20 a 35 moderada e 36 a 63 grave (Cunha, 2001).

\section{Procedimentos}

A partir de uma solicitação ao Serviço de Tecnologia da Informação do 
hospital, foram obtidos dados demográficos referentes à idade, gênero, escolaridade e tempo de internação. Estes dados foram resgatados do prontuário eletrônico e transpostos para uma planilha do programa Excel.

Níveis de depressão foram mensurados através do Inventário Beck de Depressão (BDI) e resgatados do laudo do prontuário eletrônico dos pacientes. Os dados foram dispostos em um banco no Statistical Package for Social Science for Windows (SPSS) 16.0, com o qual se obteve a freqüência e o percentual de cada variável. Ressalta-se que os dados foram obtidos retrospectivamente e os participantes não foram submetidos a procedimento ou entrevista além daquelas intrínsecas à assistência.

\section{Aspectos Éticos}

O presente estudo integra o projeto intitulado "Caracterização da demanda de avaliação psicodiagnóstica e implementação de gerenciamento e fluxos no contexto de um hospital universitário", registrado na Plataforma Brasil e aprovado pelo Comitê de Ética em Pesquisa da instituição na qual foi desenvolvido, sob o número 17-0367. Esse estudo foi projetado conforme as Diretrizes e Normas Regulamentadoras de Pesquisas Envolvendo Seres Humanos de acordo com a Resolução do Conselho Nacional de Saúde nº66 de 2012. As pesquisadoras assinaram o Termo de Compromisso para Utilização de Dados da Instituição, comprometendo-se a preservar a privacidade dos pacientes cujos dados foram analisados, já as informações e dados foram analisados retrospectivamente.

\section{Resultados}

Nos anos de 2016 e 2017, ocorreram 107 internações na Unidade de Epilepsia, sendo 7 reinternações. Ou seja, 100 pacientes foram videomonitorizados nestes dois anos, sendo que 7 foram videomonitorizados em dois momentos. $\mathrm{O}$ 
objetivo das internações era exploração diagnóstica de epilepsia refratária. A média de tempo de internação foi de 9.81 dias.

Dos 100 pacientes, 92 foram internados pelo Sistema Único de Saúde (SUS), encaminhados do ambulatório do hospital. Destes 92 pacientes, 71 tiveram solicitação de exame de avaliação neuropsicológica. Os motivos para a não solicitação dos 21 restantes foram diversos, como deficiência cognitiva severa, avaliações realizadas prévias à internação, deficiência visual ou auditiva grave, ou internação com objetivo outro que não avaliar possibilidade de cirurgia.

Das 71 solicitações de exame, 4 foram impossibilitadas devido à alta do paciente antes do término da avaliação e impossibilidade do paciente de finalizar via ambulatório. Desta forma, o Serviço de Psicologia realizou 67 avaliações neuropsicológicas, sendo 29 em 2016 e 38 em 2017.

Figura 1. Faixa etária $(N=67)$
Sessenta e duas avaliações foram iniciadas e concluídas na internação e 5 foram iniciadas na internação e concluídas em ambulatório devido à alta do paciente. $\mathrm{O}$ tempo médio entre a data de internação e a data de solicitação do exame foi de 2,66 dias; e o tempo entre a data de solicitação do exame e a liberação deste no sistema foi de 11,32 dias. Ressalta-se que excluindo as 5 avaliações finalizadas em ambulatório, esta média fica em 8,95 dias.

\section{Dados demográficos}

Observou-se uma distribuição uniforme entre os gêneros, sendo $51 \%$ feminino e $49 \%$ masculino. A média de idade foi de 42 anos, sendo que $29 \%$ encontravam-se na faixa dos 50-59 anos, $24 \%$ na faixa dos $30-39$ anos e $21 \%$ na faixa entre 40 e 49 anos, conforme Figura 1. Quanto à escolaridade, mais da metade não concluiu o Ensino Fundamental, conforme Figura 2. 


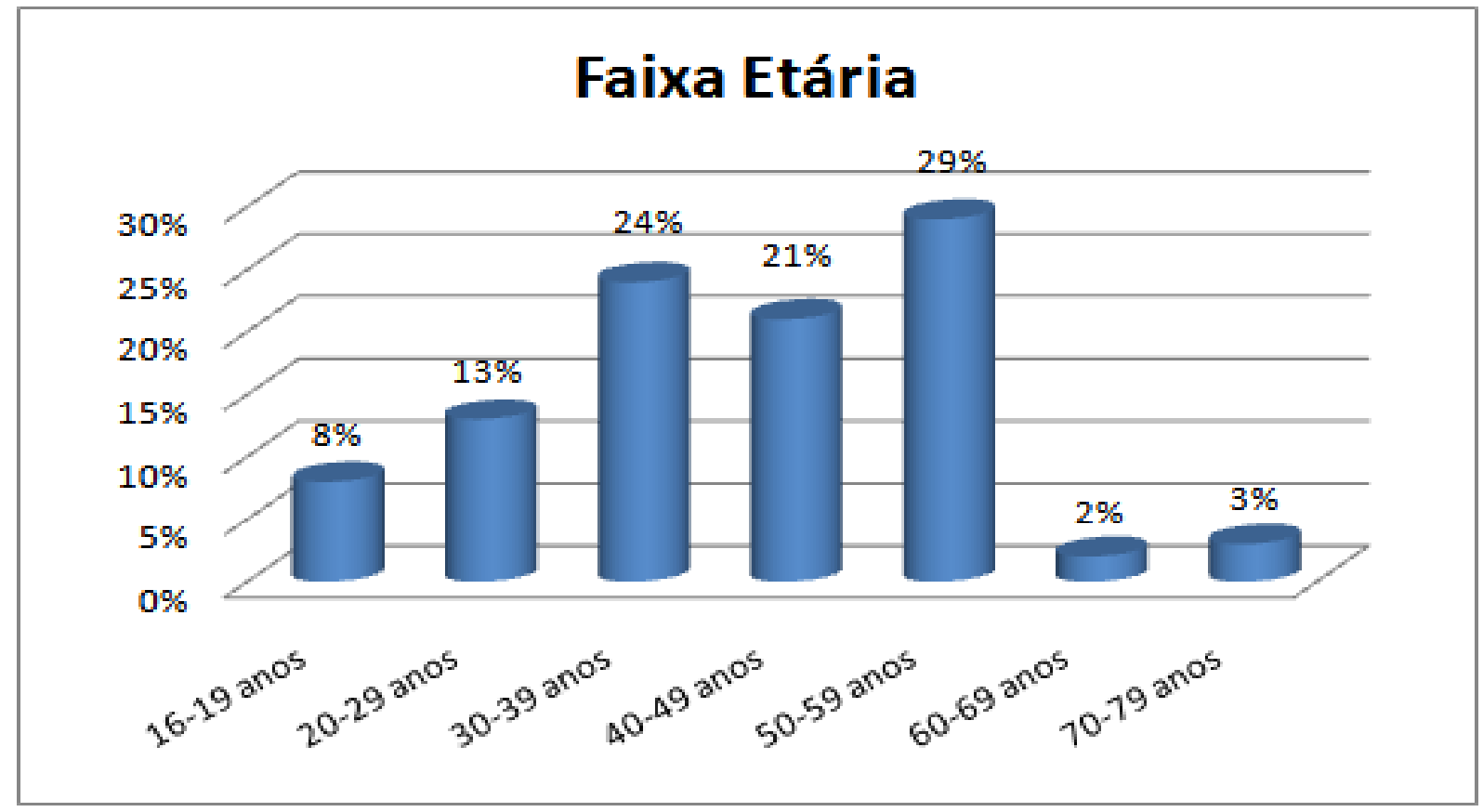

Figura 2. Escolaridade $(N=67)$

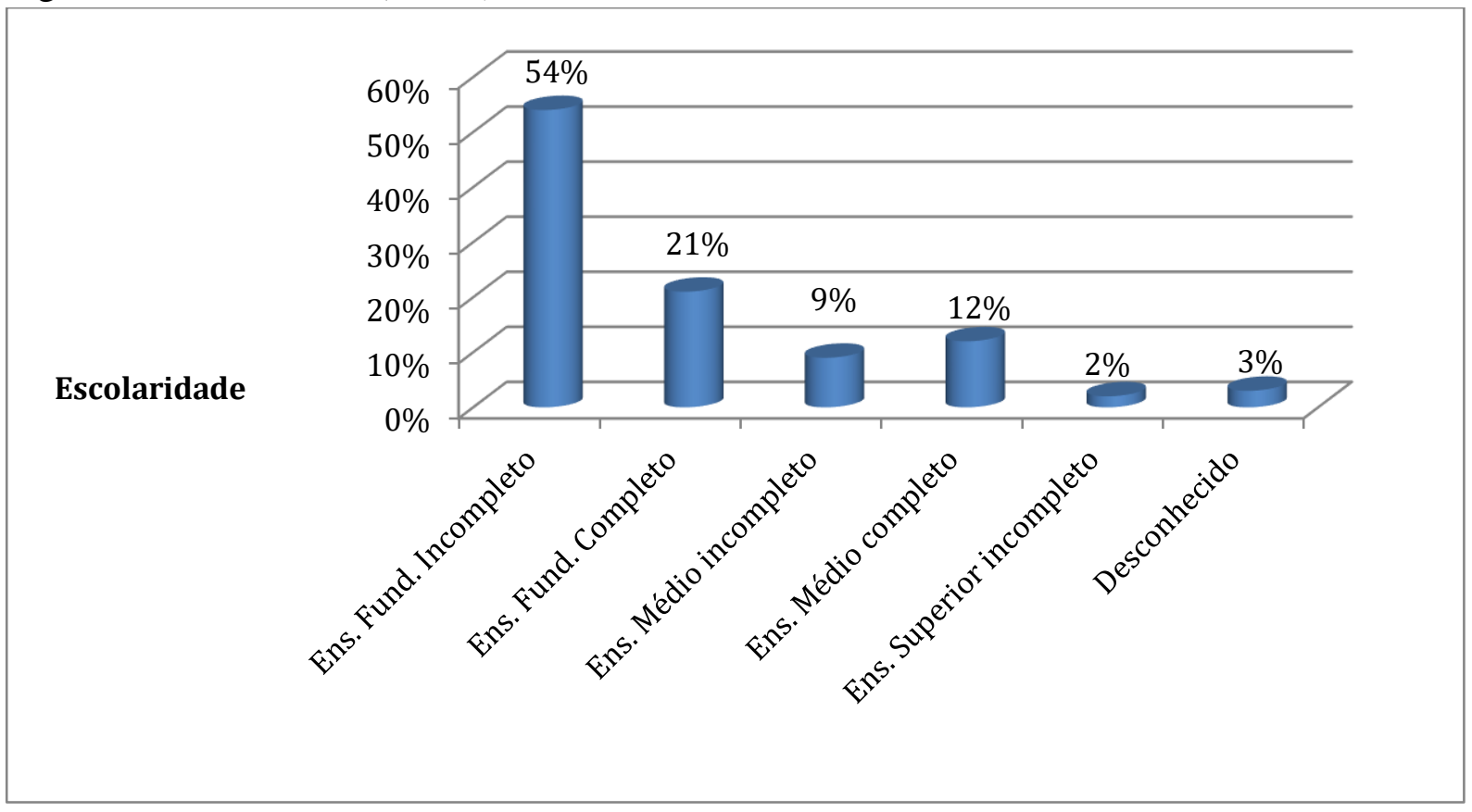

Níveis de sintomas de depressão, de acordo com BDI

Dos 67 pacientes avaliados,

cinqüenta e sete indivíduos responderam a

escala que mensura sintomas de depressão.

Seis indivíduos não compreenderam as instruções e quatro avaliações foram realizadas antes desta escala compor a bateria de avaliação neuropsicológica. Dos 57 respondentes, $63 \%$ pontuaram em 
níveis Mínimo ou Leve de sintomas de depressão; enquanto $32 \%$ da amostra pontuaram em nível Moderado e 5\% em nível Grave, conforme Figura 3.

Figura 3. Níveis de sintomas de Depressão, de acordo com BDI $(N=57)$

\section{Sintomas de Depressão}

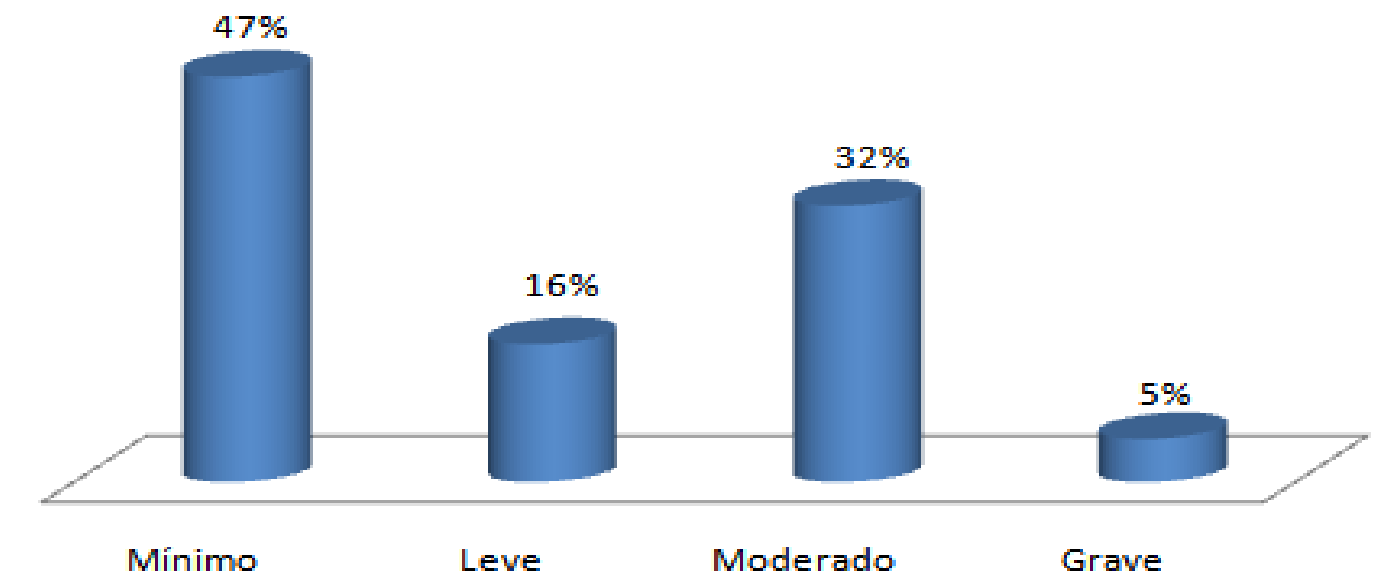

\section{Discussão}

A relação entre epilepsia refratária e

transtornos psiquiátricos vem sendo

estudada dada a alta prevalência desta comorbidade e da importância de tratar ambas as patologias para qualidade de vida desta população. Neste estudo, trinta e sete por cento dos indivíduos pontuaram o equivalente a sintomas em nível Moderado ou Grave na BDI. Este dado é inferior a outros estudos na área. Dado o elevado número de estudos na área, optou-se por selecionar estudos que considerassem somente epilepsia refratária e utilizasse a BDI na mensuração de sintomas de depressão.

O estudo de Gonçalves, Cardoso, Yasuda, \& Cendes (2017), realizado em um ambulatório de Epilepsia da Universidade de Campinas, SP entre os anos de 2008 e 2012, apontou que $77 \%$ dos indivíduos possuíam algum transtorno depressivo (Distimia, Depressão recorrente ou 
Transtorno Bipolar). Este estudo utilizou os instrumentos Mini Internacional Neuropsychiatric Interview e BDI, além de entrevista semi-estruturada, para avaliar 40 indivíduos com epilepsia mesial temporal refratária. Ainda neste centro, o estudo de Nogueira, Yasuda, Coan, Kanner e Cendes (2017), conduzido entre os anos de 2010 e 2014, com 144 indivíduos com epilepsia, indicou que $57.6 \%$ apresentavam sintomas psiquiátricos. Considerando apenas os pacientes com epilepsia refratária, esta taxa sobre para $68.3 \%$.

Garcia, Garcia-Morales e Gil-Nagel (2015) verificaram a prevalência de sintomas depressivos em um coorte de 515 pacientes com epilepsia na Espanha. Dados provenientes de diversos ambulatórios de neurologia, utilizando instrumentos Montgomery-Asberg Depression Rating Scale (MADRS) e Beck Depression Inventory-II (BDI-II), apontam que $64.8 \%$ dos pacientes com epilepsia refratária apresentam sintomas depressivos.

Há diversas causas possíveis para a discrepância entre os índices encontrados nas pesquisas prévias e neste estudo. Uma importante diferença deste estudo refere-se que foi realizado durante internação para videomonitorização com possibilidade de indicação cirúrgica. Este fato, por si só, pode representar um incremento da esperança de cura ou de melhora significativa, afetando os resultados da BDI, instrumento que considera afetos, emoções e comportamentos das últimas duas semanas. Geralmente, os pacientes internados esperaram por considerável período de tempo por esta internação, a qual pode ser considerada um elemento salvador, mitigando sentimentos e sintomas depressivos recorrentes. Os estudos trazidos foram realizados ambulatórios, sendo este o local de acompanhamento regular dos indivíduos com epilepsia.

Além disso, ressalta-se a baixa escolaridade dos participantes desta pesquisa. Este fato pode ter um impacto na dificuldade de compreensão dos itens trazidos pela escala BDI. Apesar da 
possibilidade da escala ser autoaplicável, foi percebida a necessidade de a examinadora ler para os pacientes, dada a reduzida capacidade de compreensão.

Contrastando os estudos prévios, a pesquisa multicêntrica realizada por Rocamora et al. (2018), em oito centros terciários de tratamento de epilepsia, com 49 adultos com epilepsia refratária, a taxa de depressão foi de $38.8 \%$, de acordo com a BDI. Esta realidade está mais próxima ao encontrado neste estudo.

Conclusão

A prevalência de $32 \%$ de sintomas depressivos em pacientes com ELT refratária à medicação, encontrada neste estudo, apesar de inferior a outros estudos, já é consideravelmente superior à prevalência mundial e brasileira. De acordo com o relatório global da World Health Organization (WHO, 2015), referente ao ano de 2015, é estimado que mais de 300 milhões de pessoas sofram de depressão, o equivalente a $4.4 \%$ da população mundial. No Brasil, esta taxa é de 5.8\%. Esta realidade pode ser explicada não somente por fatores culturais associados ao estigma e barreiras encontradas por pessoas com doenças crônicas, mas também à existência de caminhos patogênicos comuns entre depressão e epilepsia, propondo, desta forma, uma clara associação envolvendo diferentes neurotransmissores e alterações neurobiológicas que abrangem funcionamento psíquico e processamento emocional (Scévola et al. 2017; Torres et al., 2017).

Assim, ressalta-se que epilepsia, especialmente refratária, é um fator de vulnerabilidade para o desenvolvimento de transtornos psiquiátricos, especialmente depressão. Esta realidade aponta para a importância de um diagnóstico em tempo hábil com o objetivo de obter tratamento adequado e, assim, melhora efetiva na qualidade de vida desta população.

Observou-se elevada variabilidade na taxa de sintomas depressivos em pacientes com epilepsia refratária, oscilando entre $32 \%$ e $77 \%$ nos diferentes 
estudos revisados. Este panorama aponta

para a necessidade de mais pesquisas na área, em que população, local de coleta de dados e instrumentos utilizados sejam bem definidos e especificados. Sabe-se que a população de pacientes com epilepsia é extremamente variada, tanto em questões culturais, como da própria doença, aspectos que também influenciam desenvolvimento e formas de expressão de questões emocionais, comportamentais e psiquiátricas.

\section{Referências}

Andrade-Valença, L. P., Valença, M. M., Velasco, T. R., \& Leite, J. P. (2006). Epilepsia do lobo temporal mesial associada à esclerose hipocampal. $J$ Epilepsy Clin Neurophysiol, 12(1), 31-36. DOI 10.1590/S1676-26492006000100007.

Baxendale, S. \& Thompson, P. (2010), Beyond localization: The role of traditional neuropsychological tests in an age of imaging. Epilepsia, 51: 2225-2230. DOI 10.1111/j.1528-1167.2010.02710.x.

Cunha, J. A. (2001). Manual da versão em português das Escalas Beck. São Paulo, SP: Casa do Psicólogo.

Dias, R., Bateman, L. M., Farias, S. T., Li, C. S., Lin, T. C., Jorgensen, J., \& Seyal, M. (2010).Depression in epilepsy is associated with lack of seizure control. Epilepsy \& Behavior, 19(3), 445-447. DOI 10.1016/j.yebeh.2010.08.029.

Engel, J., McDermott, M. P., Wiebe, S., Langfitt, J. T., Stern, J. M., Dewar, S., ... \& Jacobs, M. (2012). Early surgical therapy for drug-resistant temporal lobe epilepsy: a randomized trial. Jama, 307(9), 922-930. DOI 10.1001/jama.2012.220.

Fisher, R.S., Boas W. V. E., Blume W., Elger C., Genton P., Lee P., Engel J. Jr. (2005). Epileptic seizures and epilepsy: definitions proposed by the International League Against Epilepsy (ILAE) and the International Bureau for Epilepsy (IBE). Epilepsia, 46 (4): 470472. DOI 10.1111/j.0013-9580.2005.66104.x.

Freitas-Lima, P. D., Baldoni, A. D. O., Alexandre, V., Pereira, L. R. L., \& Sakamoto, A. C. (2013). Drug utilization profile in adult patients with refractory epilepsy at a tertiary referral center. Arquivos de neuro-psiquiatria, 71(11), 856-861. DOI 10.1590/0004$282 X 20130169$.

Fuentes, D., Malloy-Diniz, L. F., de Camargo, C. H. P., \& Cosenza, R. M. (2014). Neuropsicologia: Teoria e Prática. Artmed Editora. 
Garcia, M. E., Garcia-Morales, I., \& Gil-Nagel, A. (2015). Prevalence of depressive symptoms and their impact on quality of life in patients with drug-resistant focal epilepsy (IMDYVA study). Epilepsy research, 110, 157-165. DOI 10.1016/j.eplepsyres.2014.11.003.

Gonçalves, E. B., de Oliveira Cardoso, T. A. M., Yasuda, C. L., \& Cendes, F. (2017). Depressive disorders in patients with pharmaco-resistant mesial temporal lobe epilepsy. Journal of International Medical Research, 0300060517717825.

Kanner, A. M., Byrne, R., Chicharro, A., Wuu, J., \& Frey, M. (2009). A lifetime psychiatric history predicts a worse seizure outcome following temporal lobectomy. Neurology, 72(9), 793-799. DOI 10.1212/01.wnl.0000343850.85763.9c.

Kanner, A. M., Schachter, S. C., Barry, J. J., Hersdorffer, D. C., Mula, M., Trimble, M. \& Ryvlin, P. (2012). Depression and epilepsy: epidemiologic and neurobiologic perspectives that may explain their high comorbid occurrence. Epilepsy \& Behavior, 24(2), 156-168. DOI 10.1016/j.yebeh.2012.01.007.

Kwan, P., Arzimanoglou, A., Berg, A. T., Brodie, M. J., Allen Hauser, W., Mathern, G., ... \& French, J. (2010). Definition of drug resistant epilepsy: consensus proposal by the ad hoc Task Force of the ILAE Commission on Therapeutic Strategies. Epilepsia, 51(6), 10691077. DOI 10.1111/j.1528-1167.2009.02397.x.

Laxer, K. D., Trinka, E., Hirsch, L. J., Cendes, F., Langfitt, J., Delanty, N. \& Benbadis, S. R. (2014). The consequences of refractory epilepsy and its treatment. Epilepsy \& Behavior, 37, 59-70. DOI 10.1016/j.yebeh.2014.05.031.

Nogueira, M. H., Yasuda, C. L., Coan, A. C., Kanner, A. M., \& Cendes, F. (2017). Concurrent mood and anxiety disorders are associated with pharmacoresistant seizures in patients with MTLE. Epilepsia, 58(7), 1268-1276. DOI 10.1111/epi.13781.

Orjuela-Rojas, J. M., Martínez-Juárez, I. E., Ruiz-Chow, A., \& Crail-Melendez, D. (2015). Treatment of depression in patients with temporal lobe epilepsy: A pilot study of cognitive behavioral therapy vs. selective serotonin reuptake inhibitors. Epilepsy \& Behavior, 51, 176-181. DOI 10.1016/j.yebeh.2015.07.033.

Ramos, A. A, \& Hamdan, A. C.. (2016). O crescimento da avaliação neuropsicológica no Brasil: uma revisão sistemática. Psicologia: Ciência e Profissão, 36(2), 471-485. DOI 10.1590/1982-3703001792013.

Rocamora, R., Ley, M., Molins, A., Toledo, M., Sansa, G., Bertol, V. \& Mauri, J. Á. (2018). Effect of lacosamide on depression and anxiety symptoms in patients with focal refractory epilepsy: A prospective multicenter study. Epilepsy \& Behavior, 79, 87-92. DOI 10.1016/j.yebeh.2017.10.032.

Scévola, L., Sarudiansky, M., Lanzillotti, A., Oddo, S., Kochen, S., \& D'Alessio, L. (2017). To what extent does depression influence quality of life of people with pharmacoresistant epilepsy in Argentina?. Epilepsy \&Behavior, 69, 133-138. DOI 10.1016/j.yebeh.2017.01.007. 
Torres, C. M., Siebert, M., Bock, H., Mota, S. M., Castan, J. U., Scornavacca, F., ...\& Bianchin, M. M. (2017). Tyrosine receptor kinase B gene variants (NTRK2 variants) are associated with depressive disorders in temporal lobe epilepsy. Epilepsy \& Behavior, 71, 65-72. 10.1016/j.yebeh.2017.03.030.

Valente, D. R., \& Busatto Filho, G., (2013). Depression and temporal lobe epilepsy represent an epiphenomenon sharing similar neural networks: clinical and brain structural evidences. Arquivos de Neuro-Psiquiatria,71(3), 183-190. DOI 10.1590/S0004282X2013000300011.

Valeriano-Lorenzo, E., Custodio, N., Alva-Diaz, C., Aguirre-Quispe, W., Montesinos, R., Lira, D., ... \& Cuenca-Alfaro, J. (2016). Pronóstico en cirugía de epilepsia:¿ puede influir la reserva cognitiva?. Revista de Neuro-Psiquiatría, 79(1), 31-41. DOI 10.20453/rnp.v79i1.2766.

World Health organization (WHO). Global Health Estimates. Disponível em: <http://apps.who.int/iris/bitstream/10665/254610/1/WHO-MSD-MER-2017.2eng.pdf?ua=1> Acesso em: 01 de março de 2018.

As autoras:

Juliana Unis Castan possui graduação em Psicologia pela Pontifícia Universidade Católica do Rio Grande do Sul (2006) e mestrado em Counseling and Personnel Services pela University of Maryland (2010), College Park, EUA, reconhecido pela Universidade Federal do Rio Grande do Sul (UFRGS). Atualmente, é psicóloga contratada no Hospital de Clínicas de Porto Alegre. E-mail: jcastan@hcpa.edu.br

Fernanda Rohrsetzer Cunegatto possui graduação em Psicologia pela Pontifícia Universidade Católica do Rio Grande do Sul, Brasil (2017), Programa de Aperfeiçoamento Profissional do Hospital de Clínicas de Porto Alegre E-mail: fcunegatto@hcpa.edu.br

Recebido em: 17/03/2018.

Aprovado em: 29/06/2018. 